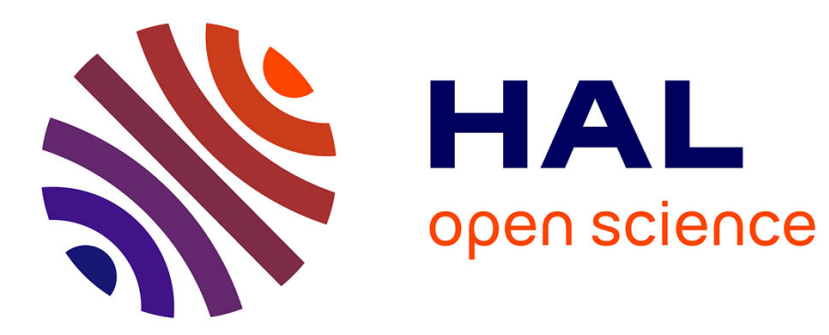

\title{
Two-wave interferences space-time duality: Young slits, Fresnel biprism and Billet bilens
}

\author{
Frédéric Chaussard, Hervé Rigneault, Christophe Finot
}

\section{To cite this version:}

Frédéric Chaussard, Hervé Rigneault, Christophe Finot. Two-wave interferences space-time duality: Young slits, Fresnel biprism and Billet bilens. Optics Communications, 2017, 397, pp.31-38. 10.1016/j.optcom.2017.03.072 . hal-01445373

\section{HAL Id: hal-01445373 \\ https://hal.science/hal-01445373}

Submitted on 24 Jan 2017

HAL is a multi-disciplinary open access archive for the deposit and dissemination of scientific research documents, whether they are published or not. The documents may come from teaching and research institutions in France or abroad, or from public or private research centers.
L'archive ouverte pluridisciplinaire HAL, est destinée au dépôt et à la diffusion de documents scientifiques de niveau recherche, publiés ou non, émanant des établissements d'enseignement et de recherche français ou étrangers, des laboratoires publics ou privés. 


\title{
Two-wave interferences space-time duality:
}

\section{Young slits, Fresnel biprism and Billet bilens}

\author{
Frédéric Chaussard ${ }^{1}$, Hervé Rigneault ${ }^{2}$, and Christophe Finot ${ }^{1, *}$ \\ ${ }^{1}$ Laboratoire Interdisciplinaire Carnot de Bourgogne, UMR 6303 CNRS-Université de \\ Bourgogne-Franche-Comté, 9 avenue Alain Savary, BP 47870, 21078 Dijon Cedex, France \\ ${ }^{2}$ Aix Marseille Université, CNRS, Centrale Marseille, Institut Fresnel UMR 7249, 13397 \\ Marseille, France \\ * Corresponding author: \\ E-mail address: christophe.finot@u-bourgogne.fr \\ Tel.: +33 380395926
}

\begin{abstract}
Taking advantage of the analogy that can be drawn between the spatial and temporal propagations, we explore two-wave temporal interference in textbook cases such as Young's double slits, Fresnel's biprism and Billet's bilens. We illustrate our approach by numerical simulations for short pulses propagating in dispersive optical fibers with parameters typical of those found in modern optical telecommunications.
\end{abstract}

Keywords: Interferences ; Fiber optics ; ultrafast optics ; time/space duality 


\section{Introduction}

Interferences of two optical waves are among the key effects used in modern optics with applications spanning from sensors development, detection of gravitational waves [1], navigation systems [2] or phase-encoded ultrafast communications [3] to cite a few. The observation of interferences has been central in the discovery of the wave nature of light [4] and famous experiments such as Thomas Young's double slits paved the debates and advances of XIX $^{\text {th }}$ century physics $[5,6]$. Young's slits are still the most famous textbook example found in physical optics courses $[7,8]$ although other devices involving two virtual sources of lights have also been developed during the XIX ${ }^{\text {th }}$ century, to confirm Young's conclusions and to increase the brightness of the resulting spatial interference pattern. In France, Augustin Fresnel (1788-1827) proposed to take advantage of a biprism $[9,10]$ whereas later, Felix Billet (1808-1882) and his assistant Jean-Baptiste-Victor Nodot, from the University of Dijon, imagined a setup made of a lens split into two parts that are slightly transversally offset [11, 12].

All those seminal experiments are essentially described in terms of space optics involving two optical waves. However, it is possible to find time domain counterparts using the well-known analogy between spatial diffraction and temporal dispersion [13-16]. This space/time duality has already been extremely fruitful and has stimulated numerous new concepts or interpretation in ultrafast optics such as temporal or spectral lenses [17-19], Talbot effect used for ultrafast sources [20], Fresnel lens [21], temporal telescope [22] or microscope [23], temporal pinhole camera [24]. Recently we used the space/time duality to demonstrate lenticular lenses [25] and to show that the Gouy phase shift has its spectral analog [19].

We propose here to explore this fascinating analogy by investigating the possibility of twowave temporal interferences as found in famous XIX ${ }^{\text {th }}$ century experiments such as Young's double slits, Fresnel's biprism and Billet's bilens. Going through these three experiments and their temporal counter parts we will highlight the physics at work, their similarity and their differences using temporal-frequency analysis. 


\section{Young's double slits}

Let us first consider the simple case where a monochromatic plane wave with wavelength $\lambda$ illuminates two identical 1D slits with aperture $A_{S}$ and separated by $S_{S}$ [see Fig. 1(a)]. In the context of Fresnel's diffraction the resulting interference pattern $I_{S}(x)$ at distance $D$, can be easily derived as:

$$
I_{S}(x, D)=\left|F^{-1}\left\{\mathcal{F}\left(U_{i n i}(x) T(x)\right) e^{-i \frac{\lambda D}{4 \pi} k_{x}^{2}}\right\}\right|^{2}
$$

where $T(x)$ is the complex transmission function that affects the input wave $U_{i n i}, x$ is the transverse coordinate and $k_{x}$ the spatial frequency, $k$ being the wave vector. $\mathcal{F}$ and $\mathcal{F}^{-1}$ are the direct and reciprocal Fourier transforms. The diffraction imprints a quadratic phase term in the momentum domain. For the sake of simplicity, we consider in the present manuscript $U_{\text {ini }}(x)=1$, i.e. the case of an incident plane wave, but other values of $U_{i n i}$ can be equally taken to extend the present discussion such as, for instance, the case of punctual sources.

In the context of Young's double slits, $T(x)$ can be expressed as

$$
T(x)=\operatorname{rect}\left(\frac{x-S_{S} / 2}{A_{S}}\right)+\operatorname{rect}\left(\frac{x+S_{S} / 2}{A_{S}}\right)
$$

where $\operatorname{rect}(x)$ is the gate function. For a sufficiently large distance $D(D>>\lambda)$, a non-localized spatial interference pattern appears with a typical interfringe distance $\Lambda_{\mathrm{S}}$ that linearly depends on $D$ and can be calculated from a usual optical path length difference [8]:

$$
\Lambda_{S}=\frac{D \lambda}{S_{S}}
$$

Let us now consider a similar situation in the time domain. Thanks to advances in phase and intensity modulators (Fig. 1(b1)), an initial continuous wave can be temporally shaped with a transmission function $T(t)$ into a sequence of two pulses of duration $A_{T}$ separated by $S_{T}$. As the counterpart of spatial diffraction found in Young's double slits experiment, we consider here temporal dispersion brought by a piece of single mode fiber with second order dispersion 
coefficient $\beta_{2}$. The dispersion occurring in the fiber leads to a temporal broadening of the pulses so that the temporal intensity profile can be predicted by [26]:

$$
I_{T}(t, D)=\left|F^{-1}\left\{\mathcal{F}(T(t)) e^{i \frac{\beta_{2}}{2} D(2 \pi f)^{2}}\right\}\right|^{2}
$$

where $t$ and $f$ are the temporal and spectral variables respectively and $D$ the propagation length in the fiber. Equation 4 is formally equivalent to Eq. 1 and, for a sufficiently long propagation distance $D\left(D>>L_{D}=A_{t}^{2} \Lambda \beta_{2} \mid\right)$ non-localized interference fringes appear in the time domain.

To perform simulations that correspond to a realistic situation, we consider two rectangular pulses with a duration $A_{T}=25$ ps being temporally spaced by $S_{T}=200$ ps [see Fig. 2(a)], such performance can be achieved using $40 \mathrm{GHz}$ transmission optoelectronics. Propagation in a standard single mode fiber at $1550 \mathrm{~nm}$ (C-band of telecom wavelengths) is considered with $\beta_{2}=-20 \mathrm{ps} / \mathrm{km}$. We neglect optical attenuation because of the low absorption found in telecom fiber $(0.2 \mathrm{~dB} / \mathrm{km}$ typically) such that propagation in several tens of kilometers can be studied without affecting the quality of the recorded pattern. We also neglect the effects of third order dispersion $\beta_{3}$ as the ratio $\beta_{3} / \beta_{2}$ is usually small for the fiber under investigation. 

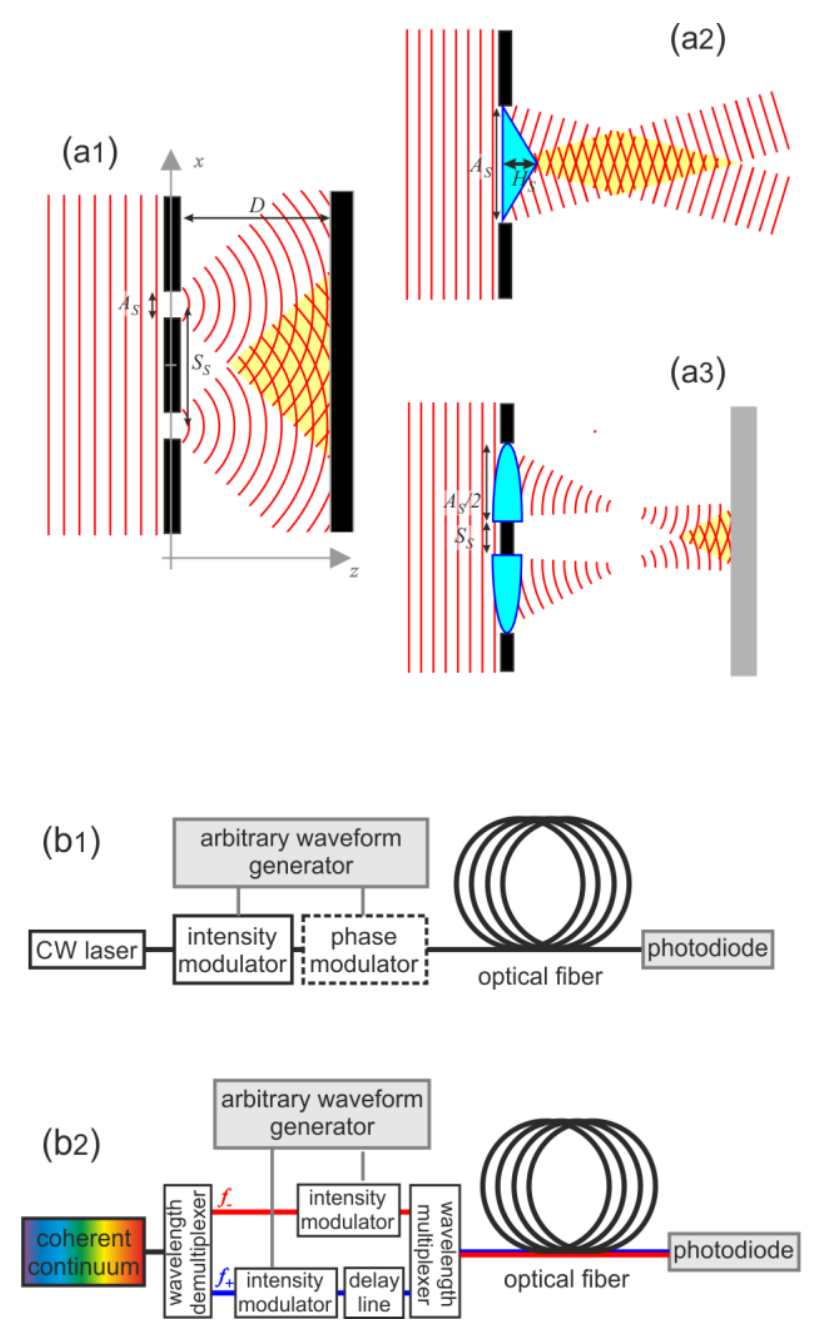

Figure 1 : (a) Usual interferometric experiments and the location of the interferences fringes : Young's double-slit experiment, Fresnel's biprism and Billet's bilens (panels 1, 2 and 3 respectively). (b) Standard telecommunication setups that may be used to create temporal interferences based on a single continuous wave that is intensity and phase modulated (b1) or using a coherent sliced continuum (b2).

Using Eq. 4, the evolution of the intensity profile of the pair of pulse (Fig. 2a) as a function the propagation distance $D$ is displayed on Fig. 2(b1). The pulses temporally expand and overlap. In the overlapping region, sinusoidal beating appears and the period of oscillation increases upon propagation [see also Fig. 3(b)]. In order to visualize this process, it is beneficial to use timefrequency representation (T-F) or spectrogram [27] that can be experimentally generated in a XFROG device [28]. This representation is provided in Fig. 3(a), the dispersion can be easily identified as spectral quadratic phase (i.e. a linear spectral chirp) and leads to a temporal 
redistribution of the energy along the temporal axis. The T-F plot of the pulses becomes then tilted with a slope $s$ being approximated in the far field by $s=1 /\left(2 \pi D \beta_{2}\right)$ [see blue dotted lines in Figs. 3(a)]. Therefore, after propagation distance $D$, and at a time $t$, two instantaneous frequencies $f$ - and $f_{+}$are present in the pulse overlapping time region and can be associated in an anomalous dispersive fiber to the leading and trailing pulses respectively:

$$
f_{ \pm}=s\left(t \mp \frac{S_{T}}{2}\right)
$$

We can therefore deduce a temporal oscillation with a frequency $\Delta f=f_{+^{-}} f_{-}=S_{T}|s|=S_{T} /\left(2 \pi D\left|\beta_{2}\right|\right)$ that is constant over the overlapping region given that the T-F plots of the pulses are parallel. We can therefore see that this temporal period of oscillation $\Lambda_{T}=2 \pi D\left|\beta_{2}\right| / S_{T}$ is in agreement with the usual interfringe expression taking into account the straightforward substitutions of $\lambda$ by $2 \pi \beta_{2}$ and $S_{S}$ by $S_{T}$. After $25 \mathrm{~km}$ of propagation (Fig. 3(a3)), temporal oscillations with a period of $15.7 \mathrm{ps}$ can be directly recorded on high speed sampling oscilloscopes with a currently available bandwidth of $70 \mathrm{GHz}$. Furthermore, the optical spectrum can be easily recorded on an optical spectrum analyser (Fig. 3c). Note that upon further propagation, the temporal pattern will become a scaled replica of the optical spectrum [29]. The present results of temporal and intensity spectral profiles do not depend on the regime of propagation in the fiber (normal or anomalous). 


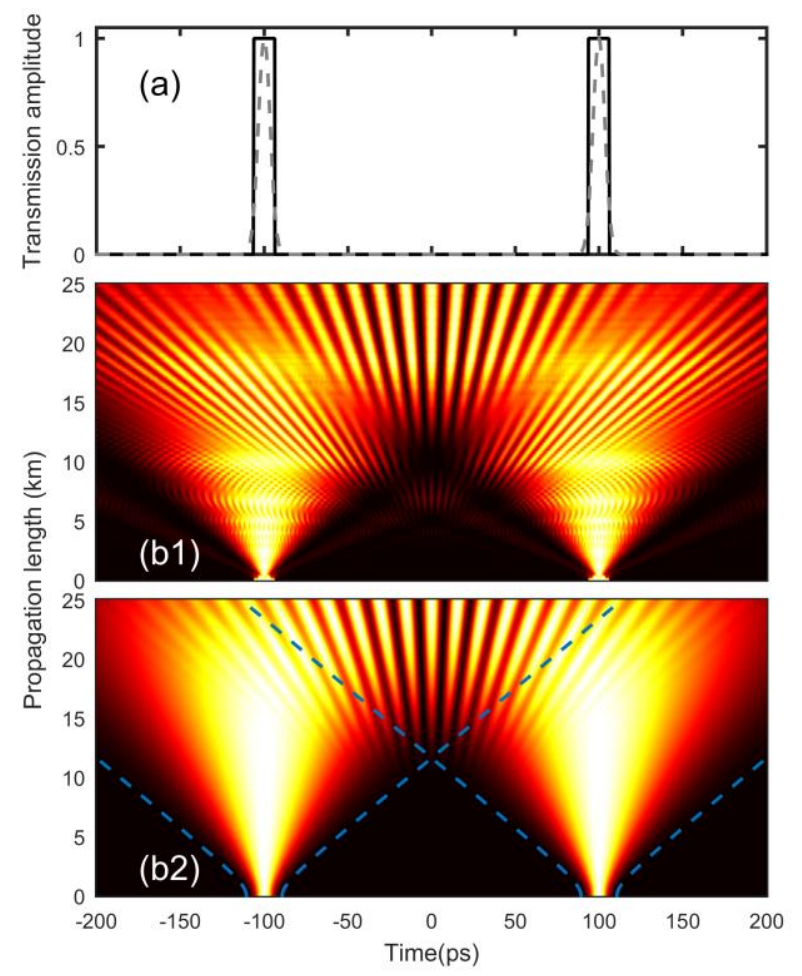

Figure 2 : (a) Temporal Young's double slits: transmission function of the intensity modulator in the case of the generation of quasi-rectangular pulses or Gaussian pulses (solid black and grey dashed lines respectively). (b) Longitudinal evolution of the temporal intensity profile in the case of rectangular temporal slits (b1) or Gaussian profiles (b2). The evolution of the temporal width a single Gaussian pulse (at $-20 \mathrm{~dB}$ ) is plotted with dashed blue line.

From experimental perspectives, it can also be interesting to evaluate the case where the initial pulses obtained after amplitude modulation are not exactly rectangular but present a smoother raise and fall edges. In Fig 2(b2), we have considered initial Fourier transform Gaussian pulses with the same full width at half maximum (fwhm) duration as the previous rectangular waveforms. In this case, temporal ripples that appear between 5 and $10 \mathrm{~km}$ for the rectangular case are not present as there are related to the sidelobes of the sinc spectrum of the rectangular case that translate, through dispersion, into temporal pedestals. We also note that whereas the rectangular waveform slightly compresses in the early stage of propagation, the Gaussian pulse undergoes a uniform temporal broadening conserving its shape. The evolution of its width can therefore be analytically calculated 
and evolves according to a $\left(1+\left|\beta_{2}\right| z / T_{0}^{2}\right)^{1 / 2}$ law where $T_{0}$ is the $1 / e$ half duration of the pulse intensity profile [26].

Let us also mention that, compared to spatial diffraction, fiber optics offers an additional degree of freedom: the dispersion can indeed be tuned and propagation in normal dispersion regime can erase the pattern resulting from propagation in the anomalous regime so that the initial waveform can be fully recovered. This forms the basis of optical dispersion-managed high speed transmissions [26]. From the practical point of view, variants of this temporal Young's double-slit experiment has found applications in microscopy with the use of spectral focusing of two coherent pulses that are frequency shifted to enable the exploration of well-defined frequencies [30]. In the presence of Kerr media, interference patterns resulting from two sources have also been investigated in the spatial domain [31], similarly to studies carried out in the temporal domain with interference and formation of shock waves $[32,33]$.
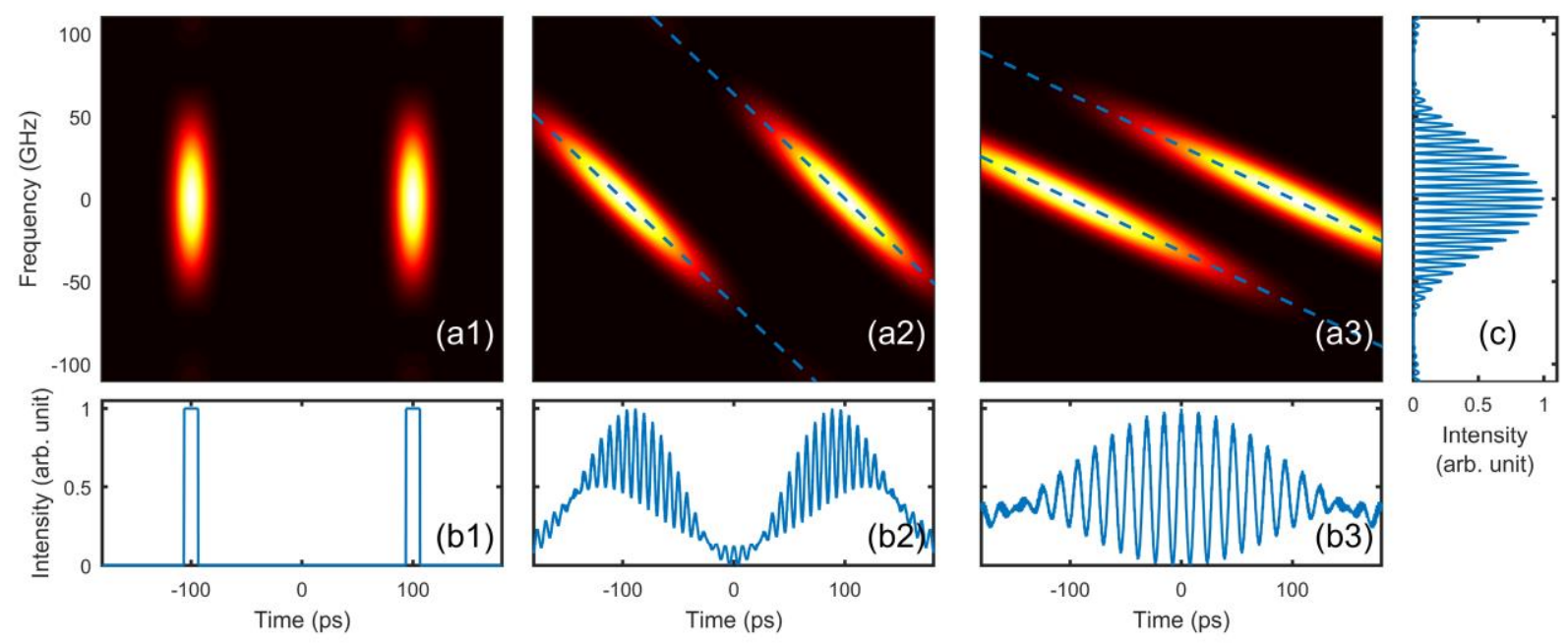

Figure 3 : Temporal Young's double slits: pulse characteristics viewed at different stages of propagation in the dispersive fiber : just after the modulation by the temporal double slits (a1), after $12.5 \mathrm{~km}$ of propagation (a2) and after $25 \mathrm{~km}$ of propagation (a3). Time-Frequency representation of the signal. Dashed blue lines represent the predictions of Eq. (4). (b) Temporal intensity profile. (c) Optical spectrum. 


\section{Fresnel's biprism}

Let us now investigate the temporal equivalent of a Fresnel's biprism. The Fresnel's biprism consists of two thin prisms joint at their bases to form an isosceles triangle. A single wavefront impinges on both prisms; the left portion of the wavefront is refracted right while the right segment is refracted left [see Fig. 1(a2)]. In the region of superposition, interference occurs since two virtual sources overlap. The Fresnel's biprism can be modeled by use of the following transmission function:

$$
T(x)=\operatorname{rect}\left(\frac{x}{A_{S}}\right) \exp \left(i(n-1) H_{S} \operatorname{tri}\left(\frac{2 x}{A_{S}}\right)\right)
$$

where $A_{S}$ is the length of the basis of the biprism, $H_{S}$ its height, and $n$ the optical index of the material. tri is the triangular function with a fwhm of $A_{S} / 2$. The temporal equivalent of this transmission function is a combination of phase and amplitude modulation [see Fig. 4(a)] :

$$
T(t)=\operatorname{rect}\left(\frac{t}{A_{T}}\right) \exp \left(i H_{T} \operatorname{tri}\left(\frac{2 t}{A_{T}}\right)\right)
$$

We consider here a large pulse $\left(A_{T}=200 \mathrm{ps}\right)$ in order to limit the impact of temporal broadening upon propagation and a maximum phase amplitude $H_{T}=10 \pi$. The triangular phase can be generated either through RF waveform generators and phase modulators or photonic generators combined with cross-phase modulation experienced during propagation in a highly nonlinear fiber [34]. 

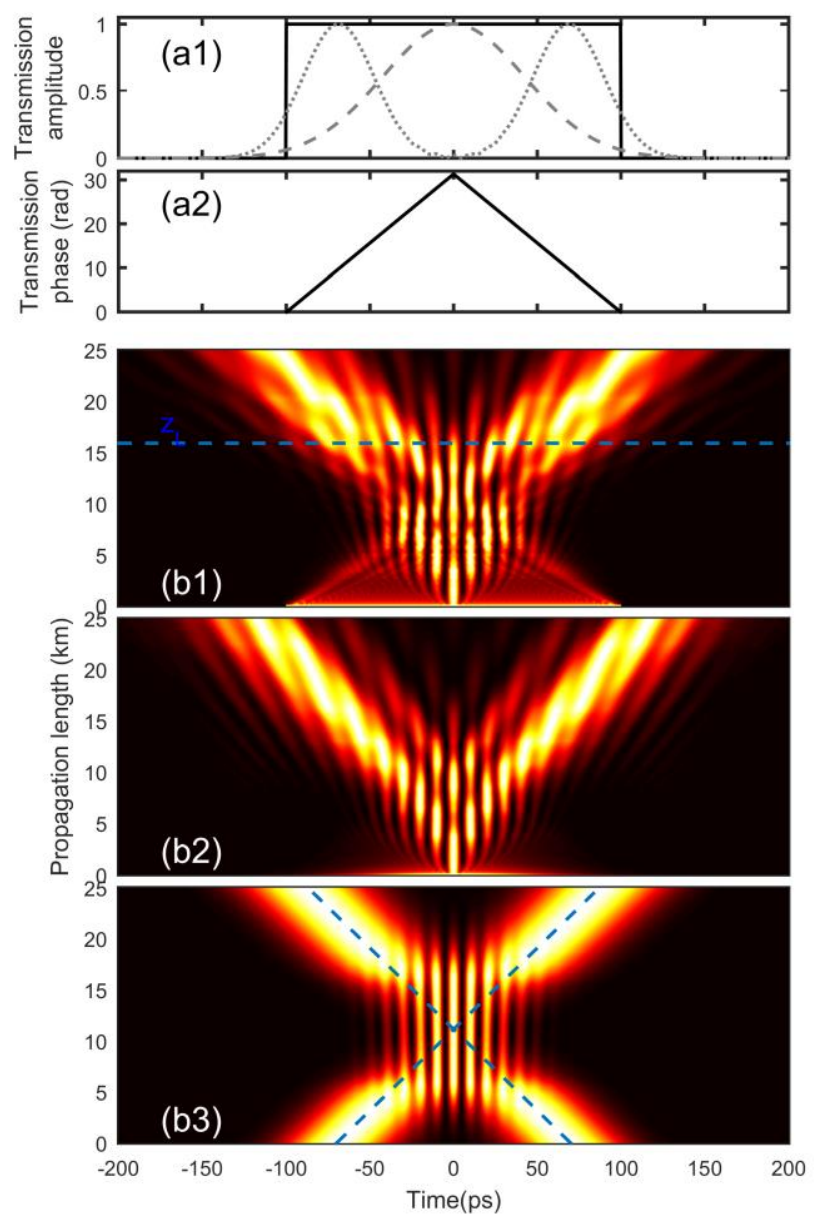

Figure 4 : (a) Temporal Fresnel's biprism: Transmission function of the intensity and phase modulators (panels a1 and a2 respectively). The direct temporal equivalent of the spatial Fresnel biprism is a quasi-rectangular long pulses modulated by triangular phase profile (solid black lines). It is compared here with a Gaussian long pulse (dash grey line) still with a triangular phase and with two transform-limited pulses (dotted grey lines) with different central frequencies and with a flat phase across the two pulses.

(b) Longitudinal evolution of the temporal intensity profile in the case of a rectangular pulse with triangular phase modulation (b1), Gaussian profile with triangular phase modulation (b2) or two transform limited pulses with different central frequencies (b3). The blue dashed lines in panel (b1) and (b3) represent the analytical prediction for $z_{L}$ and for the trajectory of the center of the Gaussian pulses respectively. 
Results of the dispersive propagation of the wave are provided in Fig. 4(b1) where we observe the resulting temporal interference pattern. Contrary to the Young's case, interferences are here localized (considering the propagation distance $D$ ). Spectrogram of Fig. 5 helps to understand the impact of the temporal biprism. The linear temporal phase modulation creates a constant frequency shift on each half of the pulse: the first half of the pulse is shifted towards $f=-H_{T} / \pi A_{T}$ whereas the other half is shifted towards the opposite frequency $f_{+}=H_{T} / \pi A_{T}$. Such property has already been exploited in ultrafast pulse processing for pulse doubling operation or frequency translation of a pulse [34-36]. Each part then travels with opposite relative speeds, the leading part travelling at a lower speed than the trailing one. This leads to a temporal overlap and the sinusoidal beating of the intensity profile [Fig. 5(b2)]. The beating frequency is here directly given by $\Delta f=2 H_{T} / \pi A_{T}$ and contrary to temporal Young's slit case, this value does not depend on the propagation distance $D$. Finally, when propagation length exceeds $z_{L}=A_{T}^{2} /\left(4\left|\beta_{2}\right| H_{T}\right)$, the $f$ - and $f_{+}$structures temporally separate and the fringes disappear. Let us note here that contrary to the temporal Young's slit case, sign of dispersion here matters and that for the present phase modulation, an anomalous dispersive fiber is mandatory. A dispersion managed system or a dispersion oscillating fiber may also provide the opportunity to observe those localized interference patterns periodically along propagation distance. 

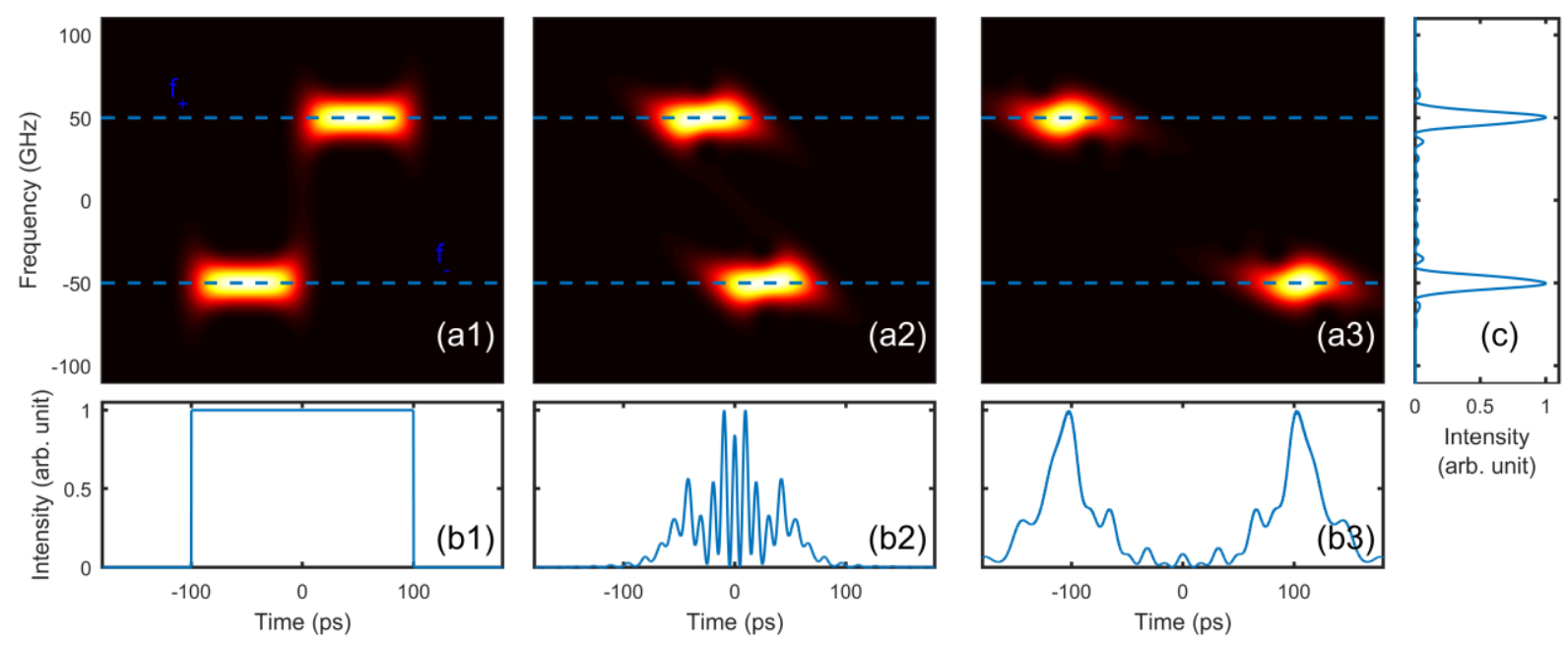

Figure 5 : Temporal Fresnel biprism: Pulse characteristics taken at different stage of propagation within the dispersive fiber: after the modulation of the Fresnel's temporal biprism, after $12.5 \mathrm{~km}$ of propagation and after $25 \mathrm{~km}$ of propagation (subplots 1, 2 and 3 respectively) (a) Time-Frequency representation of the signal. The blue dashed line represents the frequencies $f_{+}$and $f$.. (b) Temporal intensity profile. (c) Optical spectrum.

Instead of a rectangular intensity profile, it can also be interesting to test another pulse shape such as a Gaussian waveform with a fwhm temporal duration of 100 ps [see Fig. 4(a1), dashed grey line]. Results are plotted in Fig. 4(b2) and show similar trends as observed for the rectangular time modulation. Another interesting configuration that is experimentally accessible with fibers optics is to replace the linear phase modulation that shifts the instantaneous frequency of the pulses by the use of two coherent pulses with different operating wavelengths (two WDM channels spaced by $\delta f=100 \mathrm{GHz}$ with pulses with fwhm duration of $50 \mathrm{ps}$ have been used) that are time interleaved [see Fig. 4(a1), dotted line; in our example, the initial temporal spacing is $\delta T_{0}=137.5 \mathrm{ps}$ ]. Such pulses can be obtained from the frequency filtering of a continuum spectrum generated in a normally dispersive fiber [37] or in microresonators [38]. Results are displayed in Fig. 4(b3) : a similar pattern with a localized (considering the propagation distance $D$ ) zone of interferences, where the value of the interfringe, is fixed upon propagation is observed. The temporal spacing 
between the two pulses evolves linearly according to $\delta T(\mathrm{z})=\left|\delta T_{0}+2 \pi \beta_{2} \delta f\right|$. This last example grasps the physics at work and highlights the striking analogy between the temporal interferences between to pulses shifted in frequency (temporal Fresnel biprism) and the spatial interferences between two beam shifted in the k-space (spatial Fresnel biprism).

As the continuum sliced WDM sources can handle several tens of channels [38, 39], it also opens the possibility of observe the controlled interference pattern of more than 2 sources. Similarly to the spatial domain where interferences from two directions have been studied to understand the nonlinear dynamics occurring in Kerr media [40], the temporal pattern created during the collision of structures can be significantly reshaped by self-phase modulation [37, 41].

\section{Billet's bilens}

We finally investigate the possibility to extend Billet's bilens scheme to temporal imaging. A Billet's bilens is a lens of aperture $A_{S}$ and focal distance $f_{S}$ that is split into two parts that are spatially offset by a quantity $S_{S}$. The transmission function that can be associated to this device is therefore the following:

$$
\begin{aligned}
T(x) & =\operatorname{rect}\left(\frac{\left.x-\left(A_{S} / 2+S_{S}\right) / 2\right)}{A_{S} / 2}\right) \exp \left(-i \frac{\pi}{\lambda f_{S}}\left(\frac{x-S_{S} / 2}{A_{S} / 2}\right)^{2}\right) \\
& +\operatorname{rect}\left(\frac{\left.x+\left(A_{S} / 2+S_{S}\right) / 2\right)}{A_{S} / 2}\right) \exp \left(-i \frac{\pi}{\lambda f_{S}}\left(\frac{x+S_{S} / 2}{A_{S} / 2}\right)^{2}\right)
\end{aligned}
$$

In the temporal domain, the impact of a spatial lens is reproduced by a parabolic phase modulation [14] so that we can use the phase and amplitude modulation plotted in Fig. 6(a) to mimic a Billet's 
bilens with a temporal aperture of $A_{T}=200 \mathrm{ps}$, a delay $S_{T}=50 \mathrm{ps}$ between the two parts of the lens and an amplitude $H_{T}=10 \pi$ rad of the phase modulation:

$$
\begin{aligned}
T(t) & =\operatorname{rect}\left(\frac{\left.t-\left(A_{T} / 2+S_{T}\right) / 2\right)}{A_{T} / 2}\right) \exp \left(i H_{T}\left(1-\left(\frac{x-S_{T} / 2}{A_{T} / 2}\right)^{2}\right)\right) \\
& +\operatorname{rect}\left(\frac{\left.x+\left(A_{S} / 2+S_{S}\right) / 2\right)}{A_{S} / 2}\right) \exp \left(i H_{T}\left(1-\left(\frac{x+S_{T} / 2}{A_{T} / 2}\right)^{2}\right)\right)
\end{aligned}
$$

The parabolic phase modulation can be imprinted by using an arbitrary waveform generator or by use of cross-phase modulation induced by parabolic shaped pulses [42]. Results of the dispersive propagation are plotted on Fig. 6(b1) where we can observe the non-localized fringes typical of the Billet's interferences. Corresponding spectrograms are plotted in Fig. 7 and differ from the Fresnel's biprism case. After the initial modulation stage, a leading structure is linearly chirped with a chirp slope of $s_{i n}=H_{T} / 4 \pi A_{T}^{2}$ and contains only negative instantaneous frequencies. The central frequency of this part is shifted by $-H_{T} S_{T} / 4 \pi A_{T}^{2}$. On the contrary, the trailing structure exhibit only positive instantaneous frequencies. Under the action of anomalous dispersion, the slope of the chirp evolves upon propagation. In a first stage of propagation, the structures temporally compress (they are temporally focused) whereas their temporal centers also evolve towards the same direction with opposite relative velocities. Then the structures broaden and temporally separate. As the rate of temporal broadening is higher than the evolution of their temporal separation, an interference pattern is maintained. Similarly to the Young's double slit case, the period of the oscillations of the temporal profile depends on the propagation distance and linearly increases. 

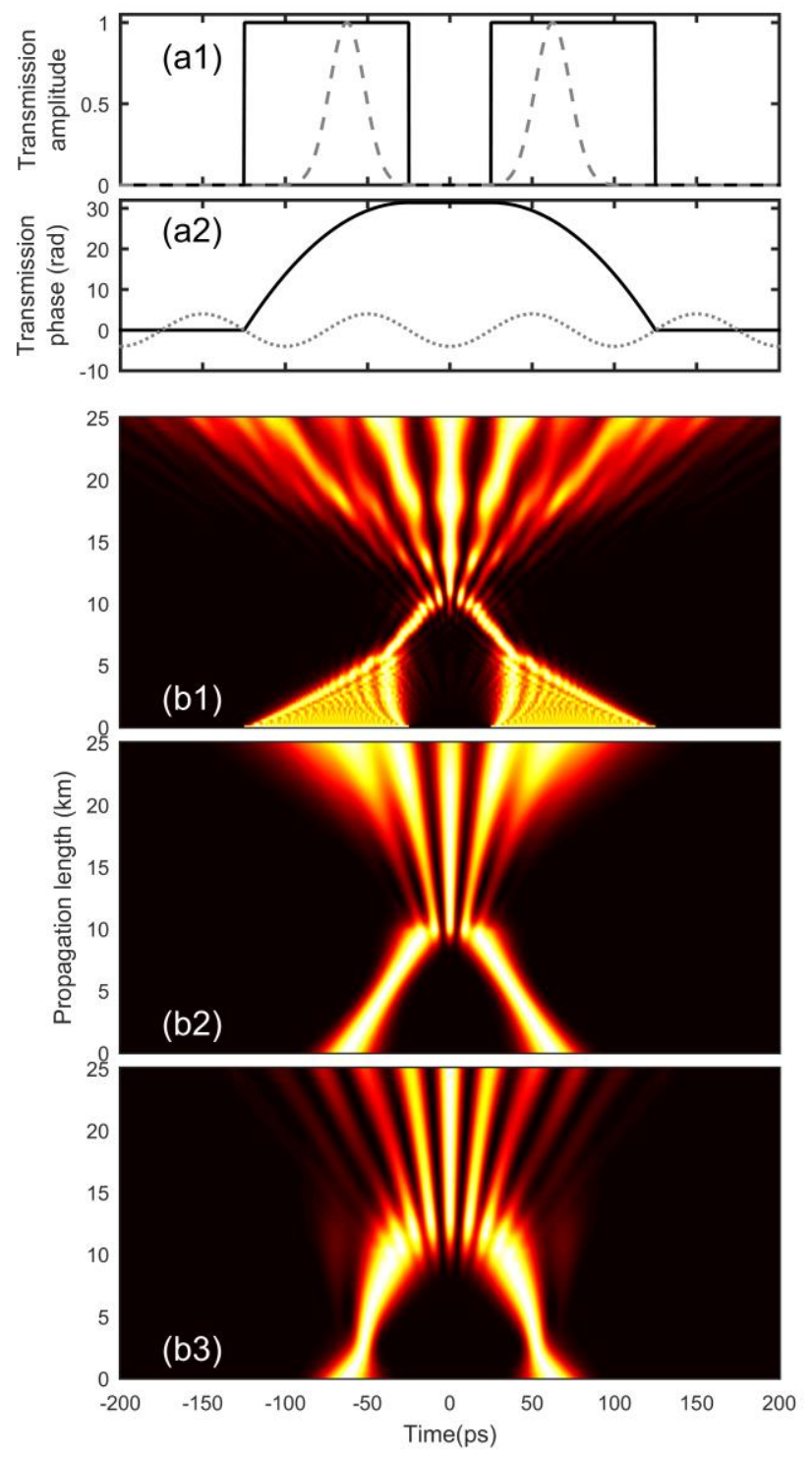

Figure 6 : Temporal Billet's bilens: (a) Transmission function of the intensity and phase modulators (panels a1 and a2 respectively). The case of two quasi-rectangular pulses modulated by quadratic phase (solid black lines) is compared to the use of two Gaussian pulses (dash grey line) with a parabolic phase modulation and to the use of two Gaussian pulses having a sinusoidal phase modulation (dotted grey lines).

(b) Longitudinal evolution of the temporal intensity profile in the case of two rectangular pulses with parabolic phase modulation (b1), two Gaussian profiles with parabolic phase modulation (b2) or two Gaussian profiles with a sinusoidal phase modulation having a peak-to-peak amplitude of 8 rad and a frequency of $10 \mathrm{GHz}(\mathbf{b 3})$. 
Once again, it can be interesting to see if similar dynamics can be reproduced when considering a pair of Gaussian pulses with the same central wavelength but delayed by 125 ps [see Fig. 6(a1) dashed line]. The same parabolic phase is applied here. We note in Fig. 6(b2) that the same interference pattern as found in the rectangular case is reproduced. What can also be of experimental interest is to evaluate the possibility of replacing the parabolic phase modulation by a sinusoidal one. Indeed, a sinusoidal modulation is much easier to achieve as it requires less optoelectronics bandwidth and can be delivered by a widely available electric clock. As a sinusoid can be approximated in the vicinity of its maximum by a parabolic fit, this explains why sinusoidal phase modulation has often been involved in temporal lenses [17, 18]. Results obtained with Gaussian pulses used in combination with sinusoidal phase are summarized in Fig. 6(b3) and stress that, despite the temporal aberrations that a non-perfect parabolic modulation may induce, a similar interference pattern is observed. We can also note here that the amplitude of the required phase modulation is significantly reduced.
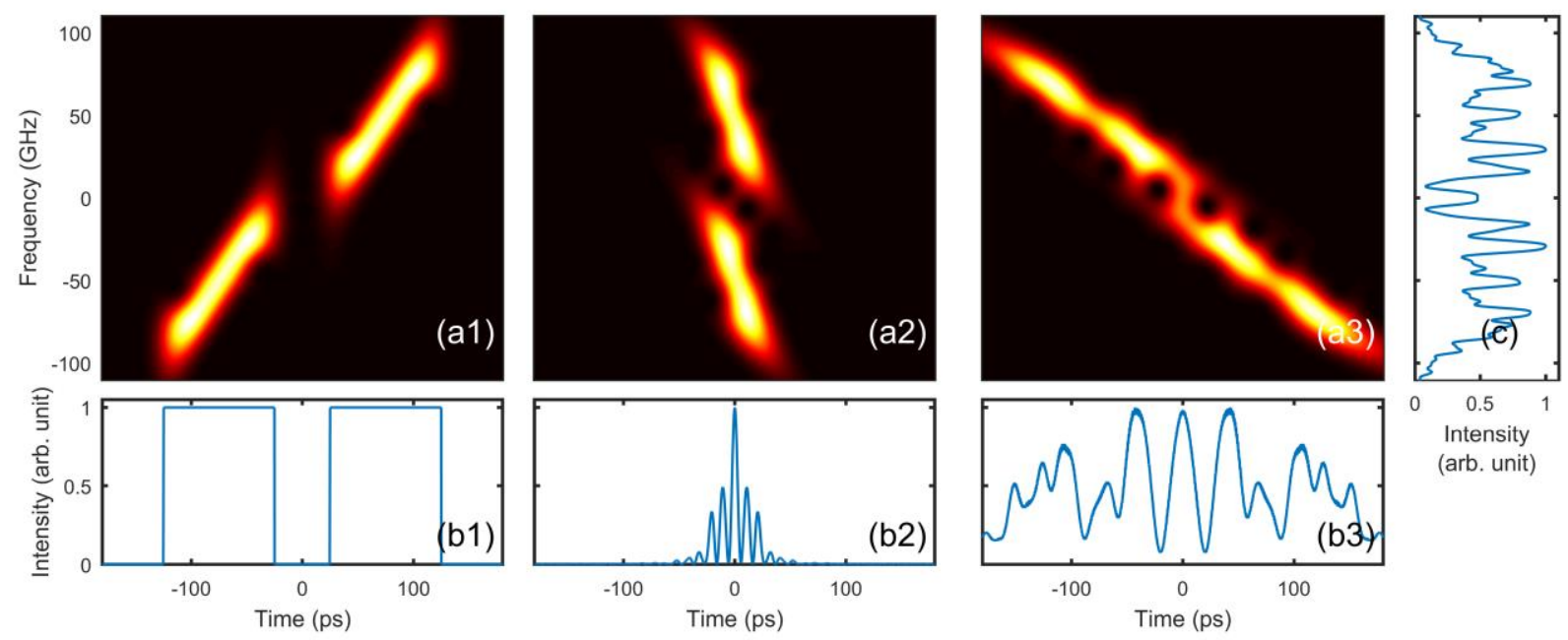
(arb. unit)

Figure 7 : Pulse characteristics taken at different stages of propagation in the dispersive fiber: after the modulation of the Billet's temporal bilens, after $12.5 \mathrm{~km}$ of propagation and after $25 \mathrm{~km}$ of propagation (subplots 1,2 and 3 respectively) (a) Time-Frequency representation of the signal. (b) Temporal intensity profile. (c) Optical spectrum. 


\section{Conclusions}

Taking advantage of the analogy that can be drawn between spatial and temporal propagations, we have explored temporal equivalent of the Young's double slits, Fresnel's biprism and Billet's bilens that are three well-known two-wave interference cases. Using devices and technologies from optical telecommunications, we have simulated these experiments using as a key ingredient the dispersive propagation experienced in kilometer long standard optical fibers. Taking advantage of time-frequency representations, we have provided a clear interpretation of the temporal interference processes at work and we have numerically demonstrated temporal interferences in Young, Fresnel and Billet cases. For the sake of simplicity, we have based our discussion on incident plane waves, but the proposed approach could be readily extended to point sources by considering chirped input conditions. We have also checked numerically that other alternative such as Meslin experimental scheme made of longitudinally offset half-lenses [7, 43] could be reproduced in the temporal domain.

The progress of arbitrary waveform generators associated with the increasing bandwidth of the optoelectronics detection enable the synthesis of phase and intensity waveforms with a higher complexity in a reconfigurable manner, similarly to the spatial light modulators for the control of diffraction pattern. The association of these novel optoelectronic modulation tools together with optical fibers appears as a flexible and viable approach to extend to the temporal domain the abundant literature dealing with the applications of two-wave interferences. 


\section{Acknowledgements}

We acknowledge the financial support of the Conseil Regional de Bourgogne (Pari Photcom), the funding of the Labex ACTION program (ANR-11-LABX-01-01). 


\section{References}

[1] T. Accadia, Virgo: a laser interferometer to detect gravitational waves, Journal of Instrumentation, 7 (2012) P03012.

[2] R. Anderson, H. Bilger, G. Stedman, " Sagnac" effect: A century of Earth-rotated interferometers, American Journal of Physics, 62 (1994) 975-985.

[3] K.-P. Ho, Phase-Modulated Optical Communication Systems, Springer US2005.

[4] R.J. Weiss, A brief history of light and those that lit the way, World Scientific1996.

[5] T. Young, The Bakerian Lecture: Experiments and Calculations Relative to Physical Optics, Philosophical Transactions of the Royal Society of London, 94 (1804) 1-16.

[6] T. Young, A course of lectures on natural philosophy and the mechanical arts. By Thomas Young, Printed for J. Johnson, London :, 1807.

[7] M. Born, E. Wolf, Principles of optics: electromagnetic theory of propagation, interference and diffraction of light, CUP Archive2000.

[8] E. Hecht, Optics, 4th, International edition, Addison-Wesley, San Francisco, (2002).

[9] Cuvres complètes d'Augustin Fresnel, Imprimerie impériale, Paris, 1866-1870.

[10] V.I. Stepanov, Auguste Fresnel. The 150th anniversary of his death, Journal of Applied Spectroscopy, 27 (1977) 825-830.

[11] F. Billet, Traité d'optique physique, Mallet-Bachelier Libraire, Paris, 1858.

[12] F. Billet, Mémoire sur les deux demi-lentilles d'interférences, Annales de Chimie et de Physique, LXIV (1862).

[13] P. Tournois, J. Vernet, G. Bienvenu, Sur l'analogie optique de certains montages électroniques: Formation d'images temporelles de signaux électriques, CR Acad. Sci, 267 (1968) 375-378.

[14] B.H. Kolner, Space-time duality and the theory of temporal imaging, IEEE J. Quantum Electron., 30 (1994) 1951-1963.

[15] J. van Howe, C. Xu, Ultrafast optical signal processing based upon space-time dualities, J. Lightw. Technol., 24 (2006) 2649-2662.

[16] R. Salem, M.A. Foster, A.L. Gaeta, Application of space-time duality to ultrahigh-speed optical signal processing, Advances in Optics and Photonics, 5 (2013) 274-317. 
[17] B.H. Kolner, M. Nazarathy, Temporal imaging with a time lens, Opt. Lett., 14 (1989) 630632.

[18] A.A. Godil, B.A. Auld, D.M. Bloom, Picosecond time-lenses, IEEE J. Quantum Electron., 30 (1994) 827-837.

[19] E.R. Andresen, C. Finot, D. Oron, H. Rigneault, Spectral Analog of the Gouy Phase Shift, Phys. Rev. Lett., 110 (2013) 143902.

[20] J. Azaña, Temporal self-imaging effects for periodic optical pulse sequences of finite duration, J. Opt. Soc. Am. B, 20 (2003) 83-90.

[21] L. Chantada, C.R. Fernández-Pousa, M.T. Flores-Arias, C. Gómez-Reino, Focusing of a train of pulses in phase opposition through a linear dispersive medium, J. Opt .A., 7 (2005) 767. [22] M.A. Foster, R. Salem, Y. Okawachi, A.C. Turner-Foster, M. Lipson, A.L. Gaeta, Ultrafast waveform compression using a time-domain telescope, Nat. Photonics, 3 (2009) 581-585.

[23] P. Suret, R. El Koussaifi, A. Tikan, C. Evain, S. Randoux, C. Szwaj, S. Bielawski, Singleshot observation of optical rogue waves in integrable turbulence using time microscopy, Nat Commun, (2016) 13136.

[24] B. Li, J. Azaña, Incoherent-light temporal stretching of high-speed intensity waveforms, Opt. Lett., 39 (2014) 4243-4246.

[25] J. Nuno, C. Finot, J. Fatome, Linear Sampling and Magnification Technique Based on Phase Modulators and Dispersive Elements: the Temporal Lenticular Lens, submitted (2017) https://hal.archives-ouvertes.fr/hal-01389069.

[26] G.P. Agrawal, Fiber-Optic Communication Systems, Wiley-Interscience2002.

[27] E.B. Treacy, Measurement and Interpretation of Dynamic Spectrograms of Picosecond Light Pulses, Journal of Applied Physics, 42 (1971) 3848-3858.

[28] S. Linden, H. Giessen, J. Kruhl, XFROG - A new method for amplitude and phase characterization of weak ultrashort pulses, Phys. Stat. Sol., 206 (1998) 119-124.

[29] K. Goda, B. Jalali, Dispersive Fourier transformation for fast continuous single-shot measurements, Nature Photonics, 7 (2013) 102-112.

[30] E.R. Andresen, P. Berto, H. Rigneault, Stimulated Raman scattering microscopy by spectral focusing and fiber-generated soliton as Stokes pulse, Opt. Lett., 36 (2011) 2387-2389.

[31] W. Wan, S. Jia, J.W. Fleischer, Dispersive superfluid-like shock waves in nonlinear optics, Nat Phys, 3 (2007) 46-51. 
[32] J.E. Rothenberg, Colliding visible picosecond pulses in optical fibers, Opt. Lett., 15 (1990) 443-445.

[33] J. Fatome, C. Finot, G. Millot, A. Armaroli, S. Trillo, Observation of Optical Undular Bores in Multiple Four-Wave Mixing, Phys. Rev. X, 4 (2014) 021022.

[34] F. Parmigiani, M. Ibsen, P. Petropoulos, D.J. Richardson, Efficient all-optical wavelength conversion scheme based on a saw-tooth pulse shaper, IEEE Photon. Technol. Lett., 21 (2009) 1837-1839.

[35] A. Latkin, S. Boscolo, R.S. Bhamber, S.K. Turitsyn, Doubling of optical signals using triangular pulses, J. Opt. Soc. Am. B, 26 (2009) 1492-1496.

[36] N. Verscheure, C. Finot, Pulse doubling and wavelength conversion through triangular nonlinear pulse reshaping, Electron. Lett., 47 (2011) 1194-1196.

[37] C. Finot, G. Millot, Collisions of optical similaritons, Opt. Express, 13 (2005) 7653-7665.

[38] J. Pfeifle, M. Lauermann, D. Wegner, V. Brasch, T. Herr, K. Hartinger, J. Li, D. Hillerkuss, R. Schmogrow, R. Holzwarth, W. Freude, J. Leuthold, T.J. Kippenberg, C. Koos, Coherent data transmission with microresonator Kerr frequency combs, Nat. Photonics, 8 (2014) 375-380.

[39] F. Parmigiani, C. Finot, K. Mukasa, M. Ibsen, M.A.F. Roelens, P. Petropoulos, D.J. Richardson, Ultra-flat SPM-broadened spectra in a highly nonlinear fiber using parabolic pulses formed in a fiber Bragg grating, Opt. Express, 14 (2006) 7617-7622.

[40] P.V. Mamyshev, C. Bosshard, G.I. Stegeman, Generation of a periodic array of dark spatial solitons in the regime of effective amplification, J. Opt. Soc. Am. B, 11 (1994) 1254-1260.

[41] S. Wabnitz, C. Finot, J. Fatome, G. Millot, Shallow water rogue wavetrains in nonlinear optical fibers, Phys. Lett. A, 377 (2013) 932-939.

[42] F. Parmigiani, P. Petropoulos, M. Ibsen, D.J. Richardson, Pulse retiming based on XPM using parabolic pulses formed in a fiber Bragg grating, IEEE Photon. Technol. Lett., 18 (2006) 829-831. [43] G. Meslin, Sur des franges d'interférence semi-circulaires, Comptes rendus hebdomadaires des séances de l'Académie des sciences 116 (1893) 250-253. 